\title{
MEAN INTERPLANETARY MAGNETIC FIELD MEASUREMENT USING THE ARGO-YBJ EXPERIMENT
}

\author{
G. Aiellit ${ }^{1,2}$, C. Bacci ${ }^{3,4}$, B. Bartoli ${ }^{5,6}$, P. Bernardini $^{7,8}$, X. J. Bi ${ }^{9}$, C. Bleve ${ }^{7,8}$, I. Bolognino ${ }^{10}$, P. Branchini $^{4}$, \\ A. Budano ${ }^{4}$, S. Bussino ${ }^{3,4}$, A. K. Calabrese Melcarne ${ }^{11}$, P. Camarri ${ }^{1,2}$, Z. CaO $^{9}$, A. Cappa $^{12,13}$, R. Cardarelli $^{2}$, \\ S. Catalanotti ${ }^{5,6}$, C. Cattaneo ${ }^{10}$, P. Celio ${ }^{10}$, S. Z. Chen $^{9}$, T. L. Chen $^{14}{ }^{\text {, Y. Chen }}{ }^{9}$, P. Creti ${ }^{8}$, S. W. Cui ${ }^{15}$, B. Z. Dai ${ }^{16}$, \\ G. D’Alí Staiti ${ }^{17,18}$, Danzengluobu ${ }^{14}$, M. Dattoli ${ }^{12,13,19}$, I. De Mitri ${ }^{7,8}$, B. D’Ettorre PiazZoli ${ }^{5,6}$, M. De Vincenzi ${ }^{3,4}$, \\ T. Di Girolamo ${ }^{5,6}$, X. H. Ding ${ }^{14}$, G. Di Sciascio ${ }^{2}$, C. F. Feng ${ }^{20}$, Zhaoyang Feng ${ }^{9}$, Zhenyong Feng ${ }^{21}$, F. GaleaZzi ${ }^{4}$, \\ P. Galeotti ${ }^{12,19}$, R. Gargana ${ }^{4}$, E. Giroletti ${ }^{10}$, Q. B. Gou ${ }^{9}$, Y. Q. Guo ${ }^{9}$, H. H. He $^{9}$, Haibing Hu $^{14}$, Hongbo Hu $^{9}$, Q. HuanG ${ }^{21}$,

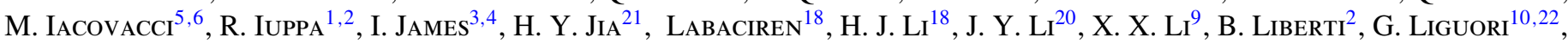

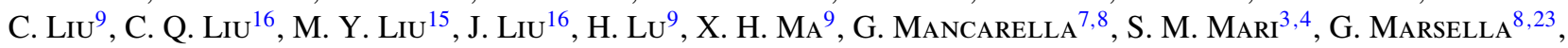 \\ D. Martello ${ }^{7,8}$, S. Mastroianni ${ }^{5}$, X. R. Meng ${ }^{14}$, P. Montini ${ }^{3,4}$, C. C. Ning ${ }^{14}$, A. Pagliaro ${ }^{17,24}$, M. Panareo $^{8,23}$, \\ L. Perrone ${ }^{8,23}$, P. Pistilli ${ }^{3,4}$, X. B. Qu ${ }^{20}$, E. Rossi ${ }^{5}$, F. Ruggieri ${ }^{4}$, L. SAgGese ${ }^{5,6}$, P. SAlvini ${ }^{10}$, R. SAntonico $^{1,2}$, P. R. Shen ${ }^{9}$, \\ X. D. Sheng ${ }^{9}$, F. Shi ${ }^{9}$, C. Stanescu ${ }^{4}$, A. Surdo ${ }^{8}$, Y. H. TAN ${ }^{9}$, P. VAllania $^{12,13}$, S. Vernetto ${ }^{12,13}$, C. Vigorito $^{12,13,19}$,

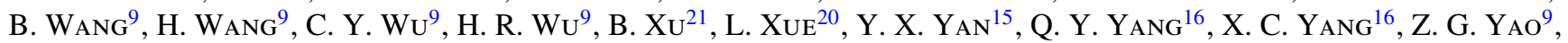

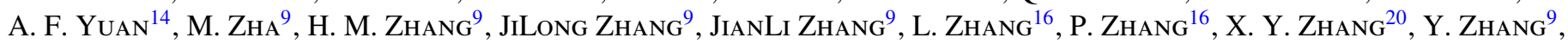

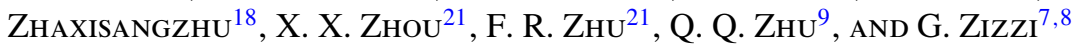 \\ (THE ARGO-YBJ COLLABORATION) \\ ${ }^{1}$ Dipartimento di Fisica dell’Università "Tor Vergata", via della Ricerca Scientifica 1, 00133 Roma, Italy \\ 2 Istituto Nazionale di Fisica Nucleare, Sezione di Tor Vergata, via della Ricerca Scientifica 1, 00133 Roma, Italy \\ ${ }^{3}$ Dipartimento di Fisica dell'Università "Roma Tre", via della Vasca Navale 84, 00146 Roma, Italy \\ ${ }^{4}$ Istituto Nazionale di Fisica Nucleare, Sezione di Roma3, via della Vasca Navale 84, 00146 Roma, Italy \\ ${ }^{5}$ Istituto Nazionale di Fisica Nucleare, Sezione di Napoli, Complesso Universitario di Monte Sant'Angelo, via Cintia, 80126 Napoli, Italy \\ ${ }^{6}$ Dipartimento di Fisica dell'Università di Napoli, Complesso Universitario di Monte Sant'Angelo, via Cintia, 80126 Napoli, Italy \\ ${ }^{7}$ Dipartimento di Fisica dell'Università del Salento, via per Arnesano, 73100 Lecce, Italy \\ ${ }^{8}$ Istituto Nazionale di Fisica Nucleare, Sezione di Lecce, via per Arnesano, 73100 Lecce, Italy \\ ${ }^{9}$ Key Laboratory of Particle Astrophysics, Institute of High Energy Physics, Chinese Academy of Science, P.O. Box 918, 100049 Beijing, China \\ ${ }^{10}$ Istituto Nazionale di Fisica Nucleare, Sezione di Pavia, via Bassi 6, 27100 Pavia, Italy \\ ${ }^{11}$ Istituto Nazionale di Fisica Nucleare, CNAF, viale Berti-Pichat 6/2, 40127 Bologna, Italy \\ 12 Istituto Nazionale di Fisica Nucleare, Sezione di Torino, via P. Giuria 1, 10125 Torino, Italy \\ ${ }^{13}$ Istituto di Fisica dello Spazio Interplanetario dell' Istituto Nazionale di Astrofisica, corso Fiume 4, 10133 Torino, Italy \\ ${ }^{14}$ Institute of Cosmic Ray Research and Department of Physics, Tibet University, 850000 Lhasa, Xizang, China \\ ${ }^{15}$ College of Physics, Science, and Information Engineering, Hebei Normal University, Shijiazhuang 050016, Hebei, China \\ ${ }^{16}$ Institute of Cosmic Ray Research and Department of Physics, Yunnan University, 2 North Cuihu Rd, 650091 Kunming, Yunnan, China \\ ${ }^{17}$ Istituto Nazionale di Fisica Nucleare, Sezione di Catania, Viale A. Doria 6, 95125 Catania, Italy \\ ${ }^{18}$ Università degli Studi di Palermo, Dipartimento di Fisica e Tecnologie Relative, Viale delle Scienze, Edificio 18, 90128 Palermo, Italy \\ ${ }^{19}$ Dipartimento di Fisica Generale dell'Università di Torino, via P. Giuria 1, 10125 Torino, Italy \\ ${ }^{20}$ Laboratory of High Energy Physics, Shandong University, 250100 Jinan, Shandong, China \\ ${ }^{21}$ Institute of Modern Physics, South West Jiaotong University, 610031 Chengdu, Sichuan, China; zhufr@ihep.ac.cn \\ ${ }^{22}$ Dipartimento di Fisica Nucleare e Teorica dell’Università di Pavia, via Bassi 6, 27100 Pavia, Italy \\ ${ }^{23}$ Dipartimento di Ingegneria dell'Innovazione, Università del Salento, 73100 Lecce, Italy \\ ${ }^{24}$ Istituto di Astrofisica Spaziale e Fisica Cosmica di Palermo, Istituto Nazionale di Astrofisica, via Ugo La Malfa 153, 90146 Palermo, Italy \\ Received 2010 September 23; accepted 2011 January 10; published 2011 February 16
}

\section{ABSTRACT}

The Sun blocks cosmic-ray particles from outside the solar system, forming a detectable shadow in the sky map of cosmic rays detected by the ARGO-YBJ experiment in Tibet. Because the cosmic-ray particles are positively charged, the magnetic field between the Sun and the Earth deflects them from straight trajectories and results in a shift of the shadow from the true location of the Sun. Here, we show that the shift measures the intensity of the field that is transported by the solar wind from the Sun to the Earth.

Key words: cosmic rays - magnetic fields - solar wind

Online-only material: color figure

\section{INTRODUCTION}

Cosmic rays from outside the solar system, mainly hydrogen and helium nuclei (Ahn et al. 2010), isotropically arrive at the Earth and can be recorded by detectors on the ground, such as the resistive plate chamber array in the ARGO-YBJ experiment (Aielli et al. 2006) at $4300 \mathrm{~m}$ above the sea level in Tibet, China. At energies around $5 \mathrm{TeV}$, the cosmic-ray arrival directions are measured by the ARGO-YBJ detector with accuracy better than $1^{\circ}$ (Iuppa et al. 2010; Aielli et al. 2010). The distribution of particle counts on the sky shows a deficit corresponding to the location of the Sun's shadow. The magnetic fields distributed in the interplanetary space (abbreviated as IMF) along the trajectories deflect these rays slightly and shift the shadow from the true location of the Sun. The Tibet $\mathrm{AS}_{\gamma}$ experiment observed the effect for the first time (Amenomori et al. 1993). With its sensitivity, year-round observation for the Tibet $\mathrm{AS}_{\gamma}$ experiment was required to make a significant Sun shadow map, therefore only the yearly variation of the shadow was reported (Amenomori et al. 2006). As a part of the magnetic field on 
the photosphere spread out to the interplanetary space by the solar wind (Severny et al. 1970; Wilcox 1971), the IMF is usually monitored using orbiting detectors only at a distance of approximately 1 AU from the Sun. Abnormally strong fluctuations of IMF could severely disturb the geomagnetic environment. What is measured using the deflection of cosmic rays is a cumulative effect along the whole path from the Sun to the Earth. Since it is strongly modulated by solar activities, IMF is better studied in quiet phases of the Sun. The observation using the ARGO-YBJ experiment described in this paper was made in such a particularly good time window when the solar activity stayed at its minimum for an unexpectedly long time since 2006.

At distances greater than 5 solar radii from the Sun center, the IMF is distributed mainly in the ecliptic plane (Ness \& Wilcox 1965; Balogh \& Jokipii 2009). Its $z$-component perpendicular to the ecliptic plane deflects cosmic rays in the east-west direction, therefore it drives the shadow with an extra shift in addition to the geomagnetic effect which constantly moves the Sun shadow toward west as observed in the moon shadow measurement (Iuppa et al. 2010). Its $y$-component, $B_{y}$, in the ecliptic plane defined to be perpendicular to the line of sight, deflects cosmic rays and thus drives the Sun shadow in the north-south direction. It has no contamination from the geomagnetic effect because the declination angle of the geomagnetic field is less than 0.5 at the ARGO-YBJ site. The measurement of $B_{y}$ is the topic of this paper.

\section{THE ARGO-YBJ EXPERIMENT DATA AND THE SUN SHADOW ANALYSIS}

The ARGO-YBJ detector is more sensitive than the Tibet $\mathrm{AS}_{\gamma}$ experiment with its fully covered active area of $5800 \mathrm{~m}^{2}$. This allows an investigation for variations of the shadow in shorter periods compared with previous observations. New phenomena associated with both spatial patterns and temporal behaviors of IMF can be observed with the ARGO-YBJ data from 2006 July to 2009 October, namely, 903 exposure days in total. Well-reconstructed events from directions within $6^{\circ}$ around the Sun are selected. They must be also within $150 \mathrm{~m}$ from the center of the array and fire at least $10056 \times 62 \mathrm{~cm}^{2}$ spatial pixels of the detector. These criteria guarantee the event reconstruction quality. Eighty-six million events survive the selection and are used for the study described here. Using this data set, a map of the Sun shadow is plotted in Figure 1, with the most significant point $(45 \sigma$, using $\mathrm{Li}-\mathrm{Ma}$ formula ( $\mathrm{Li} \&$ $\mathrm{Ma} 1983)$ for the significance estimation) located at $(0.17 \pm$ $0.02)^{\circ}$ toward north and $(0.26 \pm 0.02)^{\circ}$ toward west. In order to understand the systematic errors of the measurement, the same selection criteria are applied to events around the moon. With a significance of $55 \sigma$, similar systematic shifts of the moon shadow location are observed. The reason of the $(0.19 \pm 0.02)^{\circ}$ shift toward north is under investigation while that of $(0.31 \pm$ $0.02)^{\circ}$ toward west is exactly expected due to the geomagnetic effect (Iuppa et al. 2010).

In order to study the spatial distribution of IMF over solar longitudes, the ARGO-YBJ data mentioned above are divided into 12 groups according to the position of the Earth in terms of solar longitudes when the events are recorded. More specifically, events in each group fly along trajectories within a sector of $30^{\circ}$ in the ecliptic. In order to compensate for the Earth orbital effect, the synodic Carrington period of 27.3 days and corresponding Carrington longitudes are used to describe the position of the Earth in the sectors. Rotating together with the Sun, IMF is

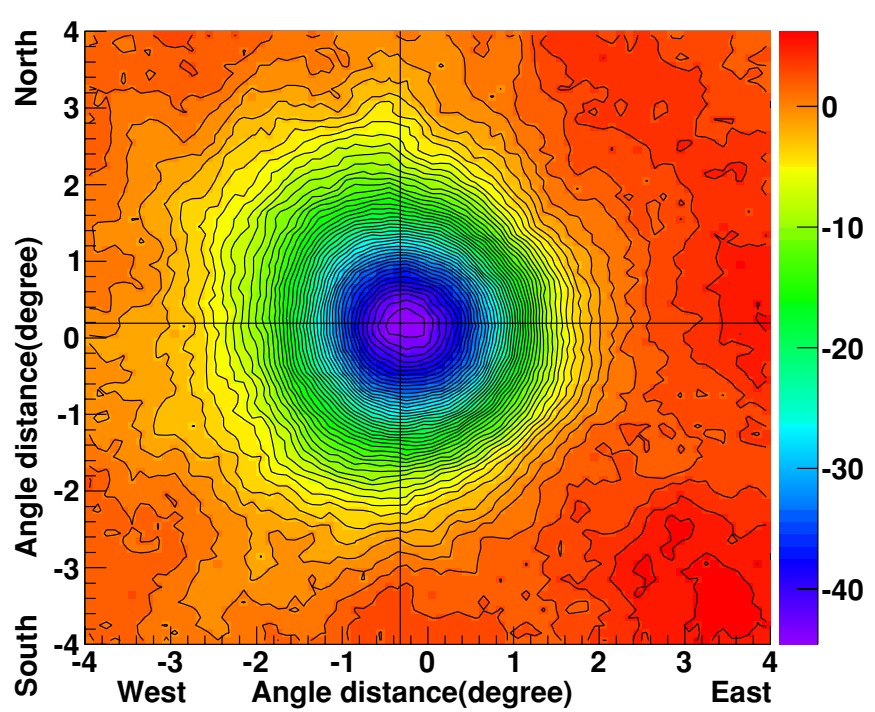

Figure 1. Sun shadow measured using all the data taken by the ARGO-YBJ experiment from 2006 July to 2009 October. This map is based on a 2D histogram on a grid of $0.1 \times 0.1$ with a smoothing within a circular bin of 1.2 as its angular radius which takes into account the point-spread function of the ARGO-YBJ detector. The central circle of the contour map indicates a significance of -43.6 standard deviation of the deficit in event count. The step between contour lines is one standard deviation. The maximum significance is -44.6 standard deviation near the cross which marks the most significant location of the moon shadow. $3.253 \times 10^{6}$ events are found in the central circular bin as the on-source event counting, while the expected background is $3.340 \times 10^{6}$.

(A color version of this figure is available in the online journal.)

nearly frozen in the sector within the 8 minutes needed for the cosmic rays to fly over the distance of $1 \mathrm{AU}$ and be deflected as well. Each group has approximately seven million events which measure the shadow with an average significance of about $10 \sigma$. The position of the shadow is measured by projecting the two-dimensional (2D) map onto the axis along the north-south direction and fitting it with a Gaussian functional form. In this way, the position is measured with an accuracy of 0.02 . A clear shift of the Sun shadow over a range greater than 0.8 from the southernmost to the northernmost position is observed as plotted in both panels of Figure 2. This clearly reveals periodical distribution patterns over solar longitudes, indicating that cosmic rays are deflected differently by IMF in the 12 sectors. The angular position of the shadow can be used as a measure of IMF as a function of the solar longitude. Since only the shadow displacement is relevant to the topic of this paper, the systematic offset on its location of 0.19 , which is found in the moon shadow observation, is removed in Figure 2.

In the upper panel of Figure 2, we used the ARGO-YBJ events collected from 2008 January to 2009 April. It is found that the shadow reaches its northernmost position at about $170^{\circ}$, then switches toward south and monotonously reaches its southernmost position at about $320^{\circ}$, and subsequently switches again toward north. This indicates that the polarization of the $y$ component of the field oscillates correspondingly. It follows that the field is spatially distributed in a bisector pattern which is also observed by the satellite-borne detectors as shown in the corresponding data which are downloaded from King \& Papitashvili (2004) ${ }^{25}$. This motivates the classification of data into the two groups, i.e., $\mathrm{G}_{1}$, which is the one under discussion, and $\mathrm{G}_{2}$, which includes all the other events. Using the $x$-component

$\overline{25}$ The corresponding data are available at http://omniweb.gsfc.nasa.gov. 

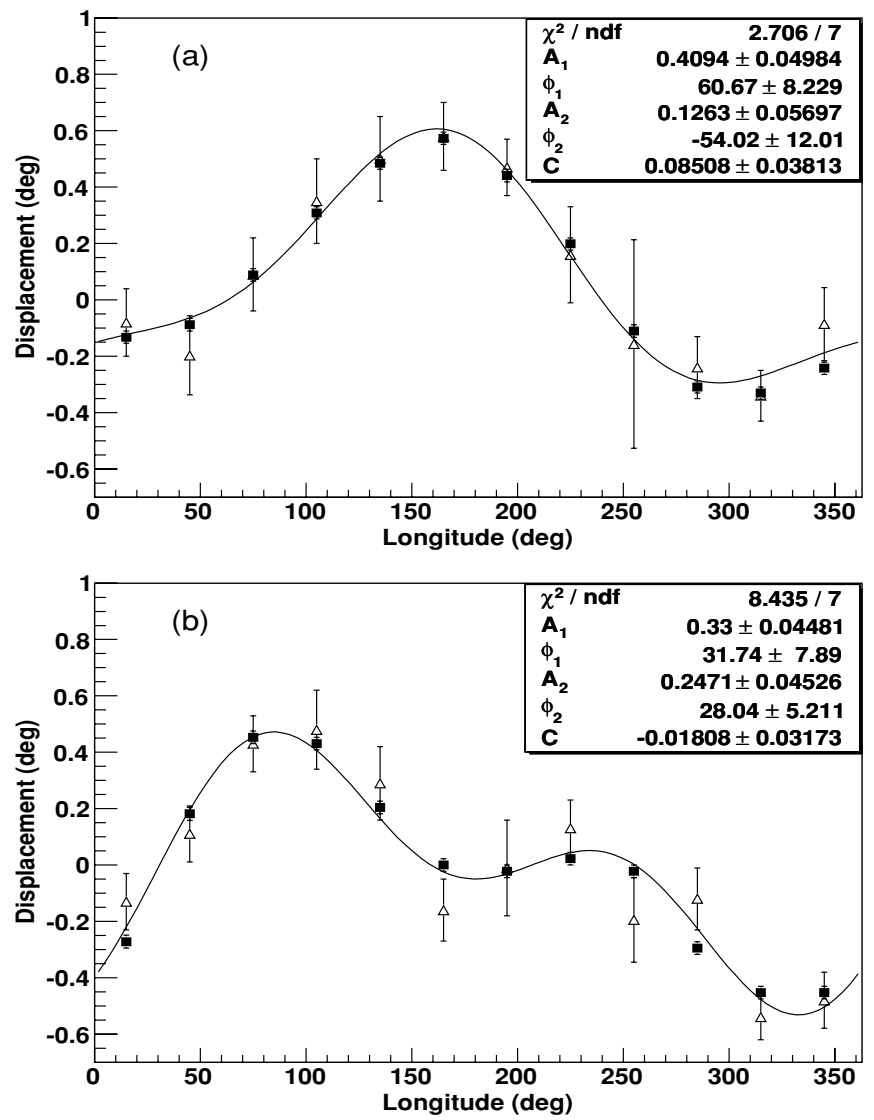

Figure 2. Shift of the center of the Sun shadow along the north-south direction during a complete Carrington period. The horizontal axis gives the Carrington longitude and the vertical axis is the angular displacement of the center of the shadow. In the upper panel (a), the observation (triangles) in the period $\mathrm{G}_{1}$ reveals that the shadow walks toward north in nearly half of the Carrington period and toward south in the rest of the period. The curve is a fit with a harmonic functional form. The squares represent the displacements of the calculated Sun shadows. In the lower panel (b), a similar shift of the shadow but with different pattern is observed in period $\mathrm{G}_{2}$, i.e., the shadow moves from side to side twice per Carrington period. $\mathrm{C}, \mathrm{A}_{i}$, and $\phi_{i}(i=1,2)$ are parameters (0th and $i$ th order coefficients and phase shifts) of the second-order harmonic functional form.

along the direction connecting the Sun and the observer, the satellites measurement of the magnetic fields clearly separates the adjacent sectors according to the polarization which points toward the Sun or away.

It is very interesting to observe a sudden switch from a 4 -sector to a simpler bisector pattern in 2007 July and a subtler return to the 4-sector pattern around 2009 April with data from King \& Papitashvili (2004). Using the ARGO-YBJ data in $\mathrm{G}_{2}$, the observed displacement of the Sun shadow is found varying in a similar 4-sector pattern as shown in the lower panel of Figure 2. Between the northernmost position at about $80^{\circ}$ and the southernmost one at about $330^{\circ}$, the shadow switches its drifting direction back and forth again between $160^{\circ}$ and $260^{\circ}$, as in a sub-period. For both cases, the spatial patterns in terms of the longitudinal distribution of the Sun shadow displacements are well fitted using harmonic functional forms as shown in Figure 2 (solid curves) and the parameters are displayed in the legends of the figures.

\section{IMF MEASUREMENT AND DISCUSSION}

Based on this observation, one can estimate the transverse component of the IMF, $B_{y}$, by fitting the measured displacement distribution as a function of the solar longitude with a minimal assumption in its model (Parker 1963; Amenomori et al. 2000). This model has been thoroughly described in Amenomori et al. (2000). The major difference is that the field has a longitudinal distribution $f(\Psi)$ that is assumed to be the same as the distribution of the displacement of the observed Sun shadow with a phase shift $\delta$. The model is briefly summarized as follows. IMF is distributed only in the ecliptic for $r>5 R_{\text {sun }}$, where $r$ is the distance from the center of the Sun and $R_{\text {sun }}$ is the solar radius. The distribution on solar longitudes is conserved along the spiral trajectories of the solar wind starting at points on the photosphere surface. The projecting direction on the photosphere is determined by the solar wind speed, $v_{r}$ (perpendicular to the surface), together with the rotating speed of the Sun, $r \omega$, where $\omega$ is the angular speed of the Sun rotation. The intensity of IMF falls as $1 / r$ along the spirals at distance far from the Sun. That is,

$$
\begin{gathered}
B_{x}=B_{0}\left(R_{\text {sun }} / r\right)^{2} f(\Psi-\delta) \\
B_{y}=B_{0}\left(R_{\text {sun }} / r\right)^{2}\left(r \omega / v_{r}\right) f(\Psi-\delta),
\end{gathered}
$$

where $B_{0}$ is the field intensity at the solar surface. $B_{0}$ and $\delta$ are the two parameters that can be estimated with our data. The solar wind that transports the fields away from the Sun has a $v_{r}$ distributed over a range from 290 to $700 \mathrm{~km} \mathrm{~s}^{-1}$. For simplicity, we assume the average value of $400 \mathrm{~km} \mathrm{~s}^{-1}$ in the analysis (King \& Papitashvili 2004).

For cosmic rays around $5 \mathrm{TeV}$, the composition has been well measured in balloon experiments (Ahn et al. 2010). A ray-tracing algorithm is developed based on this IMF model to describe particles passing by the Sun. The ray tracing is carried out with the known composition in order to reproduce the shadow effect formed by the screening of the Sun. It is found that the cosmic rays are less deflected as they fly farther from the Sun because the IMF intensity decreases as $1 / r$, however, at these larger distances there is a larger shift of the Sun shadow position due to a perspective effect. For instance, approximately $73 \%$ of the shadow shift formed by $1 \mathrm{TeV}$ cosmic rays occurs in the second half of their journey from the Sun to the Earth. Using this ray-tracing tool, it is straightforward to figure out the intensity of IMF $B_{0}$ and the phase shift $\delta$ by fitting the Sun shadow displacement data shown in Figure 2. The calculated displacements are shown in the same figure as filled squares.

The solar wind needs 4.5 days to transport IMF from the photosphere surface to the Earth where the field intensity is measured by the orbiting detectors. On the other hand, cosmicray particles take only 8 minutes to fly over the "frozen" IMF, thus the measurement by using the deflection of cosmic rays is an average over the fields along different spirals that start from different longitude regions on the photosphere surface. This is equivalent to an average over many reference points along the orbit of the Earth in a range ahead of the current Earth position in terms of Carrington solar longitudes. It results in a phase shift between the Sun shadow displacements and the IMF distribution in solar longitudes. According to the raytracing simulation, the phase shift is calculated to be $21^{\circ}$, corresponding to 1.6 days ahead. Applying this phase shift to the IMF intensities at $r=1 \mathrm{AU}$ measured by the Sun shadow displacements, we obtain measurements of $B_{y}$ as plotted in the upper and lower panels of Figure 3 for periods $G_{1}$ and $G_{2}$, respectively. The solid curves, with uncertainty represented by the shaded area, are the results of the ARGO-YBJ experiment while the solid dots represent the measurements by the orbiting 

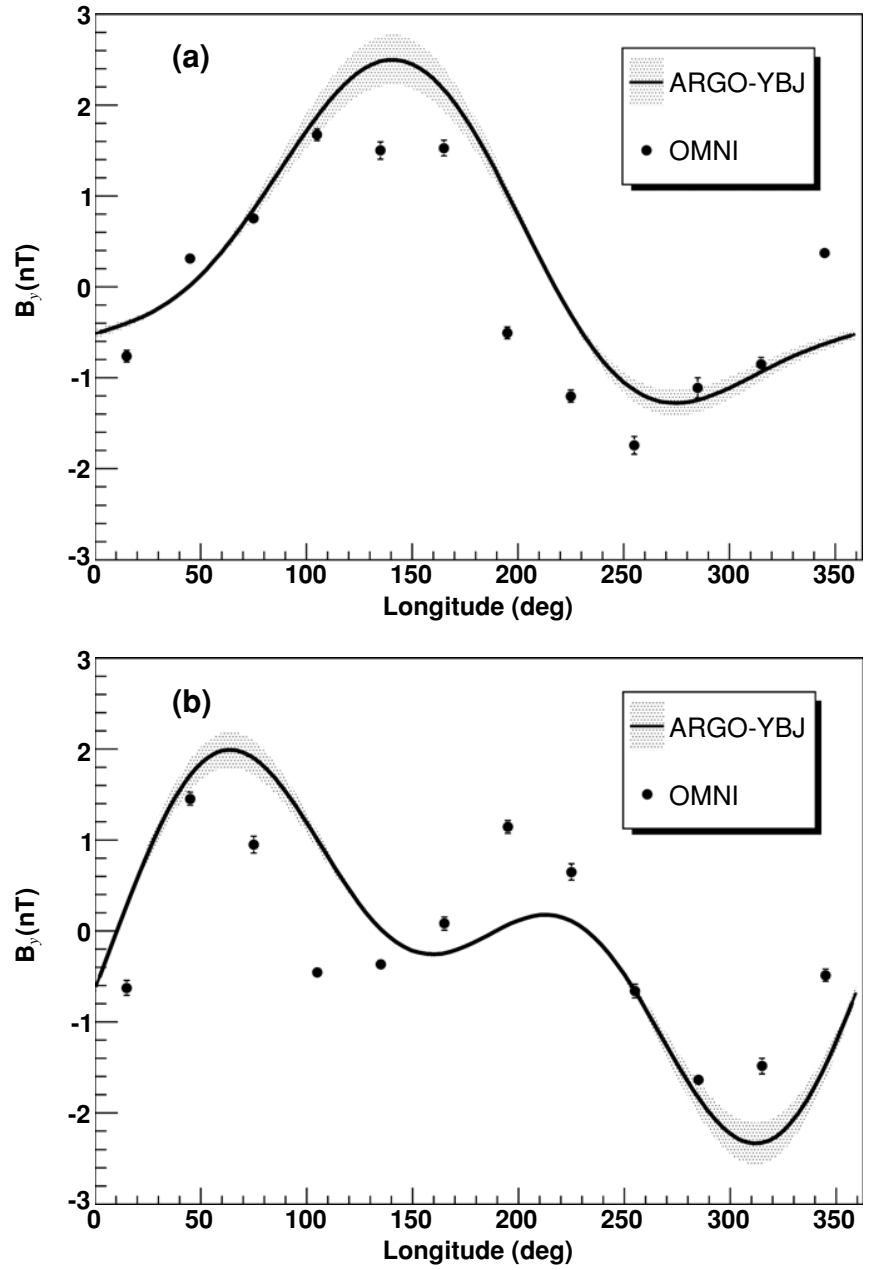

Figure 3. Comparison between two measurements of IMF using different methods. The solid curve represents the field component $B_{y}$ near the Earth measured by the ARGO-YBJ experiment using cosmic-ray deflection. In period $\mathrm{G}_{1}$ (upper panel (a)) a clear bisector pattern is observed. Positive sign indicates that the field is pointing to the center of the Sun. An uncertainty of one standard deviation is marked by the shaded area. The solid dots represent the measurements using the OMNI observational data downloaded from King \& Papitashvili (2004). In the lower panel (b), the results with the 4-sector structure in period $\mathrm{G}_{2}$ are displayed.

detectors (King \& Papitashvili 2004). The two measurements are of the same order in the amplitude of $(2.0 \pm 0.2) \mathrm{nT}$ and are consistent in the alternating periodical pattern. In order to carry out the observation in a sector of $30^{\circ}$, the current ARGO-YBJ experiment must be operated for approximately 13 complete Carrington periods to collect enough events for the required accuracy on the position of the Sun shadow, namely 0.1 , in each sector. The same folding technique with a Carrington period is used in the satellite detector data analysis as mentioned.
The measurement is particularly interesting because it refers to a "ground state" of IMF in the quiet phase, around the minimum between the $23 \mathrm{rd}$ and the 24 th solar cycles. It is also an important complement to the regular monitoring of IMF at satellite orbits because it is related to the IMF distribution over the much larger scale of $0.5 \mathrm{AU}$.

Even more importantly, this measurement could foresee fluctuations of IMF that will sweep the Earth about 2 days later, demonstrating a potential forecasting capability for magnetic storms due to solar events. Although the ARGO-YBJ detector has not sufficient sensitivity to measure the Sun shadow with the required precision in a single day, a future detector such as the LHAASO project (Cao 2010), may be sufficient to be able to measure the Sun shadow within 1 day or so. Thus, it would be practically useful in monitoring unexpectedly large shifts of the Sun shadow to foresee magnetic storms due to solar events approximately 2 days ahead of their arrival to the Earth. This triggers further study on a possible new method for space weather monitoring among many others well-developed methods (Kudela 2007). For instance, The $z$-component of IMF, which may be even more relevant to the potential forecasting, is under analysis with a careful decoupling from geomagnetic effects.

This work is supported in China by NSFC (No. 10120130794), the Chinese Ministry of Science and Technology, the Chinese Academy of Sciences, the Key Laboratory of Particle Astrophysics, CAS, and in Italy by the Istituto Nazionale di Fisica Nucleare (INFN). We also acknowledge the essential supports of W. Y. Chen, G. Yang, X. F. Yuan, C. Y. Zhao, R. Assiro, B. Biondo, S. Bricola, F. Budano, A. Corvaglia, B. D’Aquino, R. Esposito, A. Innocente, A. Mangano, E. Pastori, C. Pinto, E. Reali, F. Taurino, and A. Zerbini, in the installation, debugging, and maintenance of the detector.

\section{REFERENCES}

Ahn, H. S., et al. 2010, ApJ, 714, L89

Aielli, G., et al. 2006, Nucl. Instrum. Methods Phys. Res. A, 562, 92

Aielli, G., et al. 2010, ApJ, 714, L208

Amenomori, M., et al. 1993, Phys. Rev. D., 47, 2675

Amenomori, M., et al. 2000, ApJ, 541, 1051

Amenomori, M., et al. 2006, Adv. Space Res., 38, 936

Balogh, M. L., \& Jokipii, J. R. 2009, Space Sci. Rev., 143, 85

Cao, Z. (for the LHAASO Collaboration) 2010, Chin. Phys. C, 34, 249

Iuppa, R., et al. 2010, in Proc. 31st ICRC, arXiv:1001.0533

King, J. H., \& Papitashvili, N. E. 2004, J. Geophys. Res., 110, A02209

Kudela, K. 2007, Proc. 30th ICRC, 6, 195

Li, T. P., \& Ma, Y. Q. 1983, ApJ, 272, 317

Ness, F. N., \& Wilcox, J. M. 1965, Science, 148, 1592

Parker, E. N. 1963, Interplanetary Dynamical Process, VIII (New York: Interscience)

Severny, A. B., Wilcox, J. M., Scherrer, P. H., \& Colburn, D. S. 1970, Sol. Phys., 15,3

Wilcox, J. M. 1971, in IAU Symp. 43, Solar Magnetic Fields, ed. R. Howard (Dordrecht: Kluwer), 744 